Filamente unter der nur rudimentären Crista (welche dem Schildchen von Taxus entspricht); sie bängen frei, parallel neben einander herab oder sind selbst ein wenig gegen einander gekrümmt, sind bald gleich, bald etwas ungleich in Grösse, springen an den einander zugekehrten Seiten mit längsspalten auf und spreizen dann, in Folge Verkürzung der austrocknenden Oberfläche weit, fast horizontal auseinander.

Betreffend diese Lage der zwei Pollensäcke auf der Aussenseite des Staubfadens bei Ginkgo habe ich schon in Engler's Jahrbüchern, XXV (1897) die Ansicht ausgesprochen und begründet, dass sie sich von der ursprünglichen radiären Bildung der ältesten Sporophylle der Metaphyten (Gefässpflanzen) herleitet. Bei Welwitschia besteht noch das Ursporophyll mit arei am Gipfel des Staubfadens im Kreise angeordneten, theilweise vereinigten Pollenfächern, von denen zwei nach aussen, eines nach innen steht. Ginkgo hat davon nur die zwei äusseren Pollensäcke behalten, das innere fehlt und ist, wie sich geltend machen lässt, verloren gegangen, ausserdem hat sich wie bei allen Coniferen der Gipfeltheil des Staubblattes vegetativ als Crista entwickelt. Dagegen blieb bei Taxus die ursprïngliche radiäre Bildung erhalten, nur mit einer der Vergrösserung des vegetativen Endtheil (des Schildchens) entsprechenden Vermehrung der Sporangien auf fünf bis acht, welche ebenso rings um den Staubfaden unter dem Schildchen stehen, wie die Sporangien unter dem Schildchen des Sporophylls (Sporangiophors) von Equisetum. In der letztgenannten Gattung kann man nun bisweilen den Uebergang aus der radiären (cyklischen) Stellung der Sporangien in blos dorsale beobachten, und zwar durch Schwinden der oberseitigen Sporangien. An diaphytischen Blüten ${ }^{1}$ ) (f. proliferum), dergleichen mein Assistent Dr. Němec bei E. limosuu gefunden hat, ist dieser Vorgang sehr deutlich zu sehen.

(Schluss folgt.)

\title{
Eine Bemerkung zu J. Velenovsky's Mittheilung über eine Missbildung in den Blüten des Ranunculus acris $\mathrm{L}$.
}

Von P. Magnus (Berlin).

J. Velenovský theilt in diesem Jahrgange dieser Zeitschrift, S. 244 , mit, dass bei Politz a. d. Mettau zahlreiche Stöcke von Ranunculus acris auftraten, die nur auffallend kleine weibliche Blüten trugen. Neben ihnen traten auch normale Pflanzen mit zwitterigen Blüten gewöhnlicher Grösse auf. Die Kleinheit der weiblichen Blüte rührte hauptsächlich von der Kleinheit der Petala her. Daraus, dass die Fruehtknoten dieser weiblichen Blüten trotz der verkümmerten (wie Ve le n o v ský sagt)Corolle regelmässig zu Früchten

1) Die englischen Botaniker nennen die ungeschlechtlichen Blüten von Equisetum unpassend strobili, Zapfen. 
reifen, schliesst $V$ e le n o v ský, „dass die corollinisch entwickelte Blütenhülle nicht zur Function als 'Lockmittel für Insecten dient", weil gerade bei den weiblichen Blüten, wo „die Bestäubung durch die Insecten noch mehr nöthig wäre, die Corolle umgekehrt verkümmert".

Diese Schlussfolgerung halte ich nicht für berechtigt und muss ihr entgegentreten.

Velenorský selbst parallelisirt diese Erscheinung mit dem Auftreten der weiblichen Blüten bei Thymus. Sie ist in der That das, was wir seit Ch. Darwin Gynodiöeismus nennen. Bei vielen einheimischen Arten - ich nenne hier von Labiaten, ausser Thymus, die Salvia pratensis, bei der er vielleicht am deutlichsten auftritt; sowie Origanon vulgare, die Mentha-Arten und Glechoma hederaceum; ich nenne ferner Echium vulgare, die Dipsaceen Succisa pratensis, Scabiosa und Knautia, die Alsineen Cerastium arvense, Stellaria graminea, ferner Silene acaulis und könnte noch manche andere anführen - tritt der Gynodiöcismus in derselben Weise auf, $d$. h. es treten neben den Stöcken mit zwitterigen Blüten, Stöcke mit weiblichen Blüten auf, die immer kleinere Blumenkronen haben. Die meisten dieser Arten sind protandrisch und die Blüten der weiblichen Stöeke pflegen erst in der vorgerückten Jahreszeit zu erscheinen.

Um die biologische Bedeutung dieser Erscheinung zu verstehen, müssen wir uns vergegenwärtigen, wie sich der Besuch der Insecten vollzieht. Es ist jedem Bienenzüchter wohlbekannt, dass die Bienen beim Besuche der Blüten einer Art verharren, und es beruht darauf, dass sie Honig unterscheiden können, der aus verschiedenen Blüten gewonnen ist, z. B. Akazienhonig aus den Blüten der Robinia pseudacacia u. s. w. Ebenso verharren auch die Hummeln beim Besuche der Blüten einer Art, wie ich es wiederholt beobachtete und vom Löwenmaul (Antirrhinum majus) beschrieben habe. Sie gehen erst zum Besuche anderer Arten über, wenn die Blüten der einen Art erschöpft sind. Von den Blüten einer Art besuchen sie zuerst die Blüten mit grossen Corollen, von denen sie mehr angelockt werden, oder die sie zunächst leichter bemerken. Diese Blüten mit grossen Corollen sind eben bei den gynodiöcischen die zwitterigen Blüten, bei den diöcischen (so z. B. die ron Velenorsk $\dot{y}$ angeführten Silene Otites L. und Valeriana dicica L.) und polygamen (wie z. B. Thymus nach Velenovsky; ich habe ihn bei Berlin nur gynodiocisch bisher angetroffen) die männlichen, resp. zwitterigen Blüten. Diese werden daher zuerst besucht, und erst, wenn diese ausgebeutet sind, werden die kleineren, weiblichen Blüten von den Insecten zum Aussaugen aufgesucht und mit dem von den vorher besuchten zwitterigen, resp. männlichen Blüten mitgebrachten Pollen bestäubt. Dass die mit kleiner Corolle versebenen Blüten der weiblichen Stöcke von Ranunculus acris regelmässig Frucht ansetzen, ist also kein Beweis gegen die Function der Corolle als Anlockungsmittel für die Insecten, sondern beweist im Gegentheil, dass die 
Insecten vorher die mit grossen Corollen versehenen zwitterigen Blüten des Ranunculus acris besucht haben und sie mit dem von diesen Blüten mitgebrachten Pollen bestäubt haben. Die Kleinheit der Corolle der weiblichen Blüten ist eine Anpassung, um den späteren Besuch der weiblichen Blïten durch die Insecten zu veranlassen. Sie ist ein guter Beweis für die Function der Corolle.

Um nicht missverstanden zu werden, will ich hervorhebeu, dass ich recht wohl weiss, dass auch oft die Insecten, vorzüglich die Apiden, namentlich durch den Geruch geleitet werden. So machte mich Herr Dr. Aug. Schulz (Halle a./S.) darauf aufmerksam, dass die gewiss unscheinbaren Blüten von Thesium von zahlreichen Insecten zur Ausbeutung des reichlich abgesonderten Nectars aufgesucht werden, und ich konnte das seitdem durch eigene Beobachtung bestätigen. So dient der Farbenwechsel mancher Blüten dazu, sie in der unscheinbareren Färbung den einsichtigeren Apiden zu reserviren. So habe ich oft beobachtet, dass, wenn auf dem Lande ein Gefäss mit zuckerhaltiger Flüssigkeit, z. B. Compotreste, in einem Zimmer mit offenen Fenstern steht, die Bienen diese Flüssigkeit durch ihren Duft bald auffinden und aufsaugen. Aber dieser feine Geruch vieler Insecten hindert nicht, dass ihnen die schöne Corolle, namentlich im Freien, das Auffinden der Blüten bedeutend erleichtert, und sie auch vor allen Dingen die Blïte in der Stellung besuchen lässt, in der sie mit ihrem Körper den Pollen von der Anthere abstreifen und ihn beim Besuche anderer Blüten auf die Narben absetzen. Auch dazu dient Färbung und Gestalt der Corolle, sowie die Stellung der Nectarien.

Das Interessanteste an der Mittheilung Velenovsky's ist dieses locale Auftreten des Gynodiöcismus des Ranunculus acris L. bei Politz a. d. Mettau. Auch die Bestäubungseinrichtungen der einzelnen Arten sind nichts Starres, sondern etwas Gewordenes und Werdendes; sie sind ausgebildet und sich weiter bildend. Es ist eine dankbare Aufgabe, die Modificationen der Bestäubungseinrichtungen einer Art in verschiedenen Gebieten zu verfolgen. Während hier das gynodiöcische Auftreten einer, wie es scheint, sonst nur zwitterig beobachteten Art festgestellt ist, konnte Kirchner umgekehrt bei Stuttgart keine weiblichen Stöcke der sonst gynodiöcischen Geranium silvaticum und Knautia silvatica auffinden. Veronica officinalis fand Kirchner bei Stuttgart ausgeprägt protogynisch, während sie $H$. Müller bei Lippstadt in Westphalen homogam und Staply in England protandrisch fand. Während Prunella vulgaris und Pr. grandiflora bei Lippstadt nach H. M üller homogam sind, fand sie Kirchner bei Stuttgart in den Zwitterblüten protandrisch. Ich selbst fand bei Zermatt im Wallis Silene inflata nur gynodiöcisch, während sie bei uns in der Ebene polygam triöcisch ist. Zum interessantesten Resultate in dieser Beziehung ist Chr. A urivillius durch seine Beobachtungen gelangt, dass im Allgemeinen die Blütenpflanzen im arktischen Gebiete nicht in so hohem Grade von den Insecten abhängen, wie 
in südlichen Ländern und dem entsprechend dieselben Arten im Norden in ihrem Bestäubungsmodus von ihren Artgenossen im Süden abweichen. Zn demselben Resultate ist auch W arming bei den arktischen Cruciferen und Ericaceen gelangt. Doch scheint dies nach meinen Beobachtungen für die hohen Alpen nicht zu gelten.

Eines der bemerkenswerthesten Beispiele solcher Variation bietet, wie gesagt, das locale Auftreten des Gynodiöcismus des Ranunculus acris.

\section{Acriopsis Reinw. und ihre Stellung zu den Podochilinae.}

Von Rud. Schlechter (Berlin).

(Schluss. ${ }^{1}$ )

\section{Acriopsis javanica Reinw.}

Caespitosa, glaberrima; pseudobulbis homoblastis, ovoideis oblongisve, $2-5 \mathrm{~cm}$ longis, medio fere $1-1.5 \mathrm{~cm}$ diametientibus, 2 -3-foliatis, dimidio inferiore vaginis mox emarcescentibus primum vestitis, mox nudis; foliis lineri- vel lanceolato- vel oblongo-ligulatis, erecto-patentibus erectisve,. apice brevissime bilobulatis vel obtusis, $6-25 \mathrm{~cm}$ longis, $0 \cdot 5-2 \mathrm{~cm}$ latis; scapo gracillimo, erecto, stricto vel plus minusve flexuoso, racemoso $30-50 \mathrm{~cm}$ alto, vaginis parvulis valde dissitis obtusis ornato, racemis laxis; bracteis minutis ovatis ; sepalo intermedio lanceolato-oblongo uninervio, erecto-patente $0.4-0.5 \mathrm{~cm}$ longo, lateralibus in phyllum ovatum binervium obtusum sepalo intermedio aequilongum connatis; petalis erectopatentibus oblongo-ligulatis obtusis trinerviis, sepalo intermedio longitudine vix brevioribus, paulo tamen latioribus, labello usque ad medium columnae adnatum, e basi lineari unguiculato, in laminam patentem trilobatam dilatato, lobis lateralibus rotundatis divergentibus, intermedio producto ligulato callo obtuso elongata basin verus ornato, toto c. $0 \cdot 5-0.6 \mathrm{~cm}$ longo; columna gracili generis paulo incurva, brachiis gracilibus porrectis; elinandrio cucullato obtusato, antheram omnino oecludente; rostello triangulari acuto, anthera ovato-cordata; polliniis angustis compressis, stipite gracillimo, glandula minuta oblonga; capsula pedicellata oblonga utrinque obtusa, fide collectorum aurea, trivalvi, ea $1.5 \mathrm{~cm}$ longa, medio fere c. $0.9 \mathrm{~cm}$ diametiente, glabra.

Acriopsis javanica Reinw., in Flor. Lit. II (1825) p. 4; Bl., Bijdr. (1825) p. 377; Tabell. 71; Ldl., Orch. (1832) p. 140; Walp., Ann. VI (1860) p. 492; Hook. f., Fl. Br. Ind. VI. (1890) p. 79; Ridley, in Journ. Linn. Soc. Bot. XXXI (1896) p. 384 .

Spathoglottis? trivalvis Wall., Cat. (1830) n. 3742; Ldl, Orch. (1831) p. 120.

Acriopsis picta Ldl., in Bot. Reg. XXIX (1843) Misc. p. 105.

1) Vgl. Nr. 7, S. 245. 